\title{
Personas mayores en situación de riesgo residencial extremo en España: un diagnóstico regional
}

Irene Lebrusán. Universidad Complutense de Madrid, Madrid, España.

RESUMEN | Los beneficios de envejecer en la vivienda han sido ampliamente estudiados. No obstante, la situación residencial de las personas mayores permanece poco explorada, bajo la asunción de que sus necesidades residenciales están adecuadamente cubiertas. Este artículo analiza las condiciones en que residen las personas mayores de 65 años en España, desde una perspectiva regional comparada. La finalidad es obtener un diagnóstico residencial exhaustivo que señale qué regiones necesitan de medidas más urgentes para eliminar la infravivienda entre las personas mayores. Con base en el Censo de Población y Viviendas 2011 (último disponible), se implementan varios indicadores dirigidos a medir la vulnerabilidad residencial, la ausencia de confort, la Exclusión Residencial Ampliada y las situaciones de riesgo extremo. Los resultados confirman que existen situaciones de infravivienda con acusada desigualdad entre regiones. Además, determinados territorios registran una profunda desigualdad intraterritorial, donde quienes sufren vulnerabilidad se enfrentan a situaciones muy graves en materia residencial.

PALABRAS CLAVE | vivienda, vulnerabilidad, demografía.

ABstract | The benefits of aging in place have been widely studied. However, the housing situation of the elderly remains underexplored, below the assumption that their housing needs are adequately covered. This article analyses the housing situation of the population over 65 in Spain from a comparative regional perspective. The main purpose is to obtain an exhaustive residential diagnosis of which regions need the most urgent measures to eliminate substandard housing among the elderly. Based on the 2011 Population and Housing Census (last date available), several indicators are calculated to measure housing vulnerability, lack of comfort, extended residential exclusion and extreme residential risk situations. The results confirm marked inequality between regions regarding situations of substandard housing. Additionally, certain territories register intra-territorial inequality, where those who suffer from vulnerability face serious housing risks.

KEYwORDs | housing, vulnerability, demography. 


\section{Introducción}

La mayor esperanza de vida en salud, junto a una serie de mejoras económicas y sociales, han dado lugar a cambios reveladores tanto en el significado como en la forma de experimentar la vejez. Entre las manifestaciones de esos cambios, una de las más relevantes a nivel urbano y residencial es la que responde al deseo de permanecer de forma independiente en la vivienda y en el entorno conocido hasta edades muy avanzadas. Este hecho, analizado como proceso y como estado, se conoce en la literatura internacional como aging in place y se ha constatado tanto en España como en otros países (Costa-Font \& Mascarilla-Miró, 2009; FernándezCarro, 2013; Fernández-Carro \& Evandrou, 2014). La permanencia en el espacio conocido permitiría mantener la identidad espacial, reduciendo así el impacto de otras transiciones asociadas tradicionalmente a la vejez (la jubilación, entre otras), al no cortar con las relaciones sociales que tan importantes son en el autoconcepto (Lebrusán, 2020). En este sentido, la permanencia en la vivienda, frente a otras alternativas residenciales (institucionalización), podría ser planteada como la forma más positiva de enfrentarse a una etapa vital caracterizada por creencias negativas y para la que las personas no han sido preparadas (Rosow, 1974).

La importancia de lo anterior se incrementa por el hecho de que las personas mayores que permanecen en su domicilio tienen una vida más larga y disfrutan de mayor bienestar objetivo que las que residen en establecimientos colectivos, incluso cuando se encuentran en situación de dependencia (Barratt, 2007; Pinzón-Pulido, 2016). Por otra parte, la permanencia del mayor en su vivienda se ha constatado como una estrategia política de recorte de gastos (Office of Policy Development and Research, 2013; Oldman, 2003). No obstante, tanto estos efectos positivos como la propia permanencia dependerán, en gran medida, de la capacidad para mantener el equilibrio entre las capacidades de la persona y las demandas del entorno y la vivienda (Lawton \& Nahemow, 1973; Pynoss et al., 2003), ya que las barreras ambientales se vuelven más significativas en la vejez (Rowles, 1978). Es decir: las condiciones residenciales serán clave.

Mientras que el concepto, decisión y proceso del aging in place e incluso las pautas convivenciales asociadas ocupan un lugar importante en la literatura a nivel nacional e internacional, la atención sobre las condiciones y estado de las viviendas que ocupan las personas mayores ha sido, comparativamente, menor. Uno de los motivos podría ser la existencia de problemas residenciales más acuciantes que afectan a otros grupos, como el que retrasa la conformación de nuevos hogares y la emancipación entre los jóvenes (Echaves, 2017), las dificultades de acceso a la vivienda entre los inmigrantes (Fernández-García et al., 2018) o la inseguridad residencial asociada a otros factores sociodemográficos (Módenes, 2017). Otra causa podría ser la creencia de que la propiedad de la vivienda equivale a ausencia de problemas residenciales, siendo esta la tenencia mayoritaria entre las personas mayores: según la Encuesta de Condiciones de Vida, en un 89,8\% de los casos, los hogares encabezados por mayores de 65 años son propietarios de su vivienda (Instituto Nacional de Estadística [INE], 2019b). Un último motivo a tener en cuenta es la influencia de teorías como la expuesta en The Myth of the Golden Years 
(Gubrium, 1973), que asumen la vejez como etapa carente tanto de problemas como de deseos. En cualquier caso, la menor atención a la situación residencial de las personas mayores podría llevarnos a interpretar que en la etapa final del ciclo vital todas las necesidades potenciales se habrían podido solventar.

Como resultado, existe cierto vacío de conocimiento sobre el malestar residencial que sufren las personas mayores en España. No solo tiende a obviarse que esta situación se produce, sino que se desconoce el número de personas a las que afecta y la gravedad de las carencias. No contamos con indicadores sintéticos que midan el malestar residencial y nos permitan realizar diagnósticos en los que basar implementaciones de mejora. La motivación de este artículo radica en estas cuestiones.

$\mathrm{Al}$ analizar la situación residencial de las personas mayores en Espańa se hace necesario adoptar una perspectiva regional, como se plantea en la comprensión de la exclusión social o la pobreza española, "dado que, además de los factores estructurales ligados a las características personales y familiares, el proceso de descentralización del gasto social hacia las comunidades autónomas y las divergencias socioeconómicas entre ellas otorgan relevancia a los factores de tipo espacial" (Faura-Martínez et al., 2016, p. 60). Las diferencias espaciales se han constatado tanto en la aplicación del Índice del Desarrollo Humano simplificado (Delgado et al., 2012), como en el análisis del reparto de la pobreza (Faura-Martínez et al., 2016; Jurado \& Pérez, 2007, 2010, 2014). También aplican en materia de vivienda: el último informe que España recibió del Comité de Derechos Económicos, Sociales y Culturales (Comité DESC) de las Naciones Unidas alertaba sobre la persistencia de disparidades en materia de adecuación residencial entre comunidades autónomas (cC. AA.), señalando la ausencia de coordinación con el gobierno central como un problema ańadido (Benito, 2019). En este sentido, el análisis espacial de la presencia de situaciones de riesgo en materia de vivienda se convierte en una dimensión imprescindible para el diseño de políticas de vivienda y de integración social efectivas para las personas mayores.

En este artículo se analiza la situación residencial de las personas mayores de 65 años en España. La finalidad de esta investigación es obtener un diagnóstico residencial exhaustivo que señale qué regiones españolas necesitan de medidas más urgentes en la lucha contra la infravivienda entre las personas mayores. Como hipótesis, su situación residencial estaría caracterizada por una acusada desigualdad, en la que parte de los adultos mayores se enfrenta a graves problemas en sus viviendas. Estos serían resultado de la combinación de carencias no resueltas a lo largo del ciclo vital que tienen un impacto muy negativo sobre la calidad de vida en la vejez.

El artículo sigue el siguiente orden: el primer apartado revisa conceptos clave para la comprensión del objeto de estudio y del desarrollo del artículo. A continuación, se expone la metodología seguida y la conformación de los indicadores creados. En el epígrafe siguiente se exponen los resultados de su aplicación al territorio español y el apartado cuarto propone la posible configuración de un modelo territorial a través de la construcción del concepto-indicador de Exclusión Residencial Ampliada. Este análisis cuantitativo cierra con la profundización en las situaciones de riesgo extremo y su correspondencia territorial. Por último, se presentan las reflexiones 
finales sobre la situación residencial de las personas mayores y el propio sistema residencial español.

\section{Algunos apuntes conceptuales}

Antes de proceder al análisis, es necesario delimitar ciertas definiciones que faciliten la comprensión del objeto de estudio y de los resultados. En primer lugar, aging in place es un concepto sujeto a debate, que difiere según usuarios y contexto (Forsyth \& Molinsky, 2020) y, en ocasiones, sobreutilizado (Frank, 2001), lo que complejiza la delimitación de su contenido (véase Lebrusán, 2017). Phillips et al. (2010) lo definieron desde el marco de la gerontología social como "hacerse viejo sin moverse de casa” (p. 17), centrándose en la relación de la persona con el entorno ocupado (no institucionalizado). Esta investigación se enmarca en esta visión y, más concretamente, en la vulnerabilidad residencial como impedimento potencial para esa permanencia.

Por vulnerabilidad se entiende la exposición elevada "a determinados riesgos e incertidumbres, combinada con una capacidad disminuida para protegerse o defenderse de ellos y hacer frente a sus consecuencias negativas" (Naciones Unidas, Departamento de Asuntos Sociales y Económicos, 2003). Esta exposición al riesgo aplicado a lo residencial asume la vivienda adecuada no solo como parte del derecho básico a un nivel de vida digno (art. 11 del Pacto Internacional de Derechos Económicos, Sociales y Culturales), sino como un factor clave en la integración social. La vivienda inadecuada pondría en riesgo la integración de las personas en la sociedad, puesto que quedarían en una situación de inferioridad estructural (Cortés, 2004), de modo que las carencias en este ámbito pueden convertirse "en el factor que desencadena los procesos de exclusión” (Antón et al., 2008, p. 349). El efecto de las malas condiciones de la vivienda y de sus carencias tendrán una repercusión aún más negativa durante la vejez que en otras etapas del ciclo vital, momento en que la influencia del entorno en el bienestar es mayor (Lawton, 1977, 1983; Lawton \& Nahemow, 1973). Además, la vivienda deficiente tiene un efecto extremadamente negativo sobre la salud, especialmente en la vejez, y se asocia con mayor morbilidad (Libman et al., 2012; Navarro, 2002, 2006; Saiegh, 2012; Zuluaga, 2010).

Ayala y Navarro $(2004,2008)$ refirieron la vulnerabilidad que se sufre dentro de la vivienda como privación residencial, remitiendo para ello a la conceptualización de Sen, que lo comprendía como un funcionamiento insuficiente. En otros trabajos, Navarro (2006) se enfocaría en la exclusión residencial ampliada (diferente de la exclusión residencial extrema, que supondría la carencia de vivienda), centrándose en las situaciones de deficiencias básicas en la vivienda. Este concepto se retoma y amplía en esta investigación, donde se lo operacionaliza como la combinación de vulnerabilidad residencial y ausencia de confort. Por vulnerabilidad residencial entendemos una forma de exclusión caracterizada por la combinación de problemas residenciales de diferente gravedad que interfieren con el proceso de habitar. Las situaciones más elevadas de vulnerabilidad residencial conforman situaciones de vulnerabilidad residencial extrema. Debido al rol que cumple la vivienda en la inclusión social y en la participación en la sociedad, las personas en situación de vulnerabilidad residencial extrema estarían en situación de riesgo. 
La existencia de situaciones de exclusión residencial ampliada y de vulnerabilidad extrema estarían expresando una forma extrema de desigualdad en la sociedad espańola. Cuando esta desigualdad extrema afecta a las personas mayores, pone de manifiesto el enquistamiento de problemas a lo largo del tiempo y la incapacidad, tanto de los propios hogares como del sistema residencial, de resolver adecuadamente las necesidades en vivienda.

Respecto a la denominación de vejez, que antes referíamos como etapa renovada -que no nueva-, es necesario establecer qué comprende esta investigación por vejez y, más específicamente, su comienzo. La definición de vejez como etapa vital está sujeta a numerosas interpretaciones y diferentes enfoques (véase Lebrusán, 2017). Optaremos aquí por el umbral cronológico, estableciéndolo en los 65 años. Más allá de la facilidad estadística, el motivo fundamental es que, a pesar del retraso en la edad de jubilación (Ley 27/2011), los 65 años continúan siendo la edad mínima exigida para acceder a diferentes ayudas, beneficios y programas enmarcados en las estrategias de integración de las personas mayores y, de manera específica, a las ayudas que atañen a vivienda. Establecer el umbral de la vejez en los 65 años no niega la heterogeneidad de los componentes de este grupo etario (referido aquí como personas mayores), ni pretende reducir la complejidad de su definición, ya señalada por otros autores (Caradec, 2012).

\section{Aspectos metodológicos: la creación de indicadores y la comprensión de la exclusión residencial ampliada}

Para analizar la situación residencial de las personas mayores en España se implementan dos índices sintéticos, que nos permiten establecer la vulnerabilidad residencial y la ausencia de confort en todas las viviendas en las que resida, al menos, una persona mayor de 65 años. Antes de proceder al análisis, se comprueba mediante la aplicación de chi-cuadrado que existe relación entre el factor territorial y los indicadores, confirmando la pertinencia de su análisis (ambos indicadores presentan asociación con el factor territorial) y la desigualdad entre regiones respecto a las situaciones de riesgo.

En un segundo momento, la combinación de estos dos indicadores permite la elaboración de un clúster territorial que marcaría la Exclusión Residencial Ampliada (ERA), aportando claridad sobre la posible existencia de modelos en el sufrimiento de exclusión residencial ampliada en la vejez.

Por último, se consideran las situaciones de vulnerabilidad residencial extrema, que señalan tanto la presencia de situaciones de riesgo como la existencia de desigualdad territorial. También se indaga sobre la homogeneidad de cada territorio, a fin de conocer si existe desigualdad interna (por ejemplo, baja puntuación en la vulnerabilidad media, pero elevada presencia de personas mayores en situación de vulnerabilidad extrema).

Los indicadores creados tienen su origen en los microdatos del Censo de Población y Viviendas 2011 (INE, 20I I). Esta fuente plantea dos limitaciones: i) pérdida 
de calidad frente a censos previos; ${ }^{1}$ y ii) su antigüedad. No obstante, tras valorar todas las fuentes existentes, el Censo de Población y Hogares 2011 continúa ofreciendo la mejor información disponible. Por una parte, es la fuente que mayor número de variables residenciales ofrece, a la par que permite contrastar con datos demográficos y de hogar. Por otro lado, su tamaño muestral es mayor que el de otras operaciones estadísticas, siendo además suficientemente representativo a nivel regional, incluso tras aplicar filtros por edad. Por último, aun reconociendo su antigüedad, las dudas sobre la vigencia de los datos quedan solventadas al analizar información más reciente procedente de otras fuentes, como la que proporciona la European Union Statistics on Income and Living Conditions (EU-SILC). En estas estadísticas (con menor riqueza que el Censo y menor desagregación territorial), las condiciones residenciales no muestran una mejora que haga dudar sobre la validez de los datos utilizados. De hecho, variables como la disponibilidad de aseo o ducha dan cuenta de un empeoramiento en el periodo 2011-2019 (al respecto, véase Eurostat, 2019).

La razón de crear dos indicadores diferenciados es la posibilidad de alcanzar la máxima exhaustividad con base en los datos disponibles, analizando tanto las carencias que, desde una perspectiva etaria, conducirían a una situación de vulnerabilidad residencial, como aquellas variables que, sin ser imprescindibles, podrían contribuir a aumentar el bienestar residencial de la persona mayor: no todas las carencias tendrían la misma gravedad o impacto en otros grupos poblacionales. La decisión sobre la inclusión de las variables en cada uno de los indicadores y sobre la asignación de pesos a las categorías es resultado de un proceso exhaustivo que incluye investigación cualitativa y documental. Primero se analizaron la literatura internacional existente y diferentes estudios de caso nacionales sobre la medición de la calidad residencial. Al considerar que este análisis reflejaba problemas residenciales muy alejados de la realidad española o para los que no existían datos -véase estudios de Fernández-Mayoralas y Rojo, 2005; o de Nolan y Winston, 2011-, se optó por revisar la legislación nacional en materia de habitabilidad, que permitía marcar mínimos (cuestiones de mayor gravedad) y aterrizar ciertas cuestiones. También se consultaron informes técnicos sobre edificabilidad y estadísticas nacionales para conocer la generalización de determinadas dimensiones en las viviendas españolas. Por último, las entrevistas a arquitectos especializados en la materia, técnicos del Ayuntamiento de Madrid y personas mayores permitieron comprobar el impacto subjetivo de ciertas carencias e incluso revisar la adecuada inclusión de ciertas variables (véase análisis ampliado en Lebrusán, 2017).

El indicador de vulnerabilidad se compone de cinco dimensiones: i) habitabilidad, que refiere la existencia de dotaciones mínimas e imprescindibles (agua corriente; aseo; evacuación de aguas residuales; disponibilidad de bañera o ducha); ii) salubridad, que contiene dos variables con constatados efectos negativos en la

1 A diferencia de los censos previos, el Censo 2011 recurre a una recogida mixta de la información, a partir de una muestra de aproximadamente un $12 \%$ de la población (5,7 millones de personas, 3 millones de viviendas), que se cruzó con el Padrón y otros registros (INE, 2011). Sobre su validez, consúltese Lebrusán (2017); y sobre otras cuestiones relacionadas, Alberich (2017). 
salud (estado del edificio, ${ }^{2}$ presencia de hacinamiento); iii) dotaciones de seguridad subjetiva, como la disponibilidad de tendido telefónico (imprescindible para acceder a servicios de teleasistencia domiciliaria o "botón antipánico") y la convivencia con usos no residenciales en el edificio; ${ }^{3}$ iv) aislamiento físico, analizando la presencia de ascensor en edificios de tres plantas o más, y la accesibilidad; ${ }^{4}$ y por último, v) dotaciones de bienestar necesarias, como serían la existencia de calefacción o aparatos que permitan calentar, al menos, una estancia de la vivienda.

El índice que señala la ausencia de confort se compone de tres variables dicotómicas: i) disponibilidad de internet; ii) existencia de agua caliente central; y iii) disponibilidad de gas en el edificio, distribuido por tuberías. Es necesario señalar que estas variables no resultan imprescindibles para cubrir los parámetros de una vivienda adecuada ni marcan por sí mismas vulnerabilidad o riesgo de exclusión. No obstante, su inclusión en el análisis se considera de interés, por entenderse que su presencia incrementa el confort de la persona mayor y podría funcionar como corrector de algunos problemas menores. Así, la disponibilidad de internet podría aumentar potencialmente la integración social y se ha comprobado su impacto positivo en la reducción de la soledad en la vejez (Şar et al., 2012). Por su parte, la disponibilidad de agua caliente central señala que su uso está garantizado incluso ante ingresos reducidos, ya que las entrevistas revelaron que limitar el uso de agua caliente, incluso en los meses más fríos, era utilizado como estrategia de ahorro entre los mayores con menos recursos. Por último, la disponibilidad de gas por tuberías asegura que existe una supervisión por parte de la distribuidora y aplicación obligatoria de una normativa de seguridad. Además, según una consulta a la Asociación Profesional de Técnicos de Bomberos, el gas por tuberías reduce el riesgo de incendio doméstico frente a otras formas de calentar el hogar. Habiendo valorado estas cuestiones, se considera que su inclusión resulta útil para el diagnóstico, si bien se otorga un peso menor a su carencia.

La construcción de los indicadores sigue cuatro pasos:

a. Identificación y recodificación de las variables que van a formar parte de los indicadores;

b. Asignación de pesos - entre 0 y 1 , siendo 1 la situación más desfavorable- a cada una de las categorías de las variables seleccionadas;

c. Se calcula cada índice de manera separada; $y$

d. Se estandarizan los índices. Cuanto más se aproximen a 1, mayor malestar.

2 En el Censo 2011 esta variable adopta cuatro categorías: a) Ruinoso, si el edificio se encuentra apuntalado, existe declaración de ruina o se está tramitando; b) Malo, si existen grietas acusadas o abombamientos en fachadas, hay hundimientos o se aprecia que ha cedido la sustentación del edificio; c) Deficiente, si las bajadas de lluvia o el sistema de evacuación de aguas residuales están en mal estado, hay humedades en la parte baja del edificio o filtraciones en los tejados o cubiertas; y d) Buen estado.

3 Se incluyó esta dimensión tras comprobar, con expertos y personas mayores, que al no tener vecinos a los que acudir en caso de necesidad se incrementaba la situación de inseguridad subjetiva de la persona mayor.

4 Un edificio puede tener ascensor y no ser accesible, al presentar, por ejemplo, escaleras de acceso al ascensor. 
Tanto la inclusión de variables como sus pesos siguen los supuestos de normativa legal/necesidad/presencia. Ejemplificando la importancia de estos tres aspectos para acreditar que una vivienda cumple las condiciones mínimas para ser habitada, esta debe disponer de agua corriente en su interior (indicado en normativa legal). Además, que el agua corriente en la vivienda es una necesidad básica resulta fuera de toda discusión (necesidad objetiva). Pero si lo fuese, o surgiesen dudas acerca de su objetividad, la disponibilidad generalizada (presencia mayoritaria) de agua corriente en las viviendas españolas nos indica que es un bien extendido y valorado por los hogares como necesario. Por tanto, aquellas viviendas que disponen de este bien serán calificadas con un peso 0 , mientras que aquellas que carecen del mismo tendrían asignado un peso 1. Hay que recordar que las categorías de las variables originales son excluyentes entre sí, todas ellas categóricas. Para un análisis más detallado, puede consultarse la metodología en Lebrusán (2017).

Por su parte, la realización de entrevistas a expertos en vivienda permitió reflexionar sobre la inclusión de otras variables cuya necesidad no resultaba tan obvia en un principio, o modificar los pesos asignados. Además, la consulta a personas mayores sobre su experiencia residencial y la pertinencia de ciertas variables permiten contar con la validez del usuario, destinada a cubrir la empatía necesaria para evitar riesgo de invalidación cuando el instrumento seleccionado para medir un fenómeno resulte inadecuado para las personas a las que se les aplica (Hernández et al., 2014). Por último, una vez realizado el indicador, se recurrió a la validación externa por parte de doce expertos (dos demógrafos expertos en vejez; tres arquitectos; un aparejador especializado en rehabilitación residencial; cuatro sociólogos expertos en vivienda y vulnerabilidad; una estadística; una matemática experta en diseńo de indicadores) mediante reuniones individuales y grupales. Esta consulta y validación responde a la intención de corregir posibles sesgos o incorreciones sobre la denominada penalización de cada categoría. Las tablas siguientes (Tabla 1 y Tabla 2) son el resultado final de este proceso.

\section{TABLA I Dimensiones y pesos del Indicador de Vulnerabilidad Residencial}

\begin{tabular}{|l|l|l|c|}
\hline \multicolumn{1}{|c|}{ DIMENSIÓN } & \multicolumn{1}{|c|}{ VARIABLE } & \multicolumn{1}{c|}{ CATEGORÍAS } & $\begin{array}{c}\text { PENALI- } \\
\text { ZACIón }\end{array}$ \\
\hline \multirow{4}{*}{$\begin{array}{l}\text { DOTACIONES } \\
\text { MínIMAS } \\
\text { (HABITABILIDAD) }\end{array}$} & \multirow{2}{*}{ Agua corriente } & No tener & 1 \\
\cline { 2 - 4 } & Aseo & Abastecimiento privado (no público) & 0,3 \\
\cline { 2 - 4 } & Evacuación aguas residuales & No tener & 0,9 \\
\cline { 2 - 4 } & Baño o ducha & No tener & 0,9 \\
\cline { 2 - 4 } & & Distinta de alcantarillado & 0,3 \\
\hline \multirow{3}{*}{ SALUBRIDAD } & Estado edificio & No tener & 0,8 \\
\cline { 2 - 4 } & & Ruinoso & 0,8 \\
\cline { 2 - 4 } & Hacinamiento & Malo & 0,7 \\
\cline { 2 - 4 } & Deficiente & \\
\hline
\end{tabular}




\begin{tabular}{|l|l|l|c|}
\hline \multicolumn{1}{|c|}{ DIMENSIÓN } & \multicolumn{1}{|c|}{ VARIABLE } & \multicolumn{1}{c|}{ CATEGORÍAS } & $\begin{array}{c}\text { PENALI- } \\
\text { ZACIÓN }\end{array}$ \\
\hline \multirow{2}{*}{$\begin{array}{l}\text { DOTACIONES SEgU- } \\
\text { RIDAD PERCIBIDA }\end{array}$} & Acceso a tendido telefónico & No tener & 0,4 \\
\cline { 2 - 4 } & Tipo de usos en el edificio & Convivencia con usos no residenciales & 0,3 \\
\hline \multirow{2}{*}{ AISLAMIENTO } & Ascensor (corregido)* & No tener & 0,7 \\
\cline { 2 - 4 } & Accesibilidad & No tener & 0,7 \\
\hline \multirow{2}{*}{$\begin{array}{l}\text { DOTACIONES } \\
\text { BIENASTAR (NECE- } \\
\text { SARIAS) }\end{array}$} & Calefacción** & Individual (no colectiva o central) & 0,1 \\
\cline { 2 - 4 } & & No, pero aparatos & 0,5 \\
\cline { 2 - 4 } & No tener & 0,6 \\
\hline
\end{tabular}

* SE CONSIDERAN EDIFICIOS PLURIFAMILIARES CON MÁS DE DOS PLANTAS

** ESTA VARIABLE ASIGNA UN MENOR PESO EN CANARIAS, ANDALUCÍA, COMUNIDAD VALENCIANA Y EN CEUTA Y MELILla. LA AUSENCIA DE CALEFACCIÓN NO ES PENADA (SE LE ASIGNA VALOR

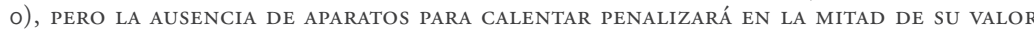
(MÁXIMA VARIABILIDAD ESTADÍSTICA) CON RESPECTO A OTRAS COMUNIDADES AUTÓNOMAS, EXCEPTO PARA EL CASO DE CANARIAS, CEUTA Y MELILLA, REGIONES PARA LAS QUE SE ELIMINA LA PENALIZACIÓN, EN CUALQUIER CASO. ESTO SE DEBE A QUE EN LAS REGIONES DE CLIMATOLOGÍA MÁS CÁLIDA EL IMPACTO QUE LA AUSENCIA DE CALEFACCIÓN TIENE SOBRE LA CALIDAD DE VIDA ES MENOR Y SE BUSCA EVITAR SOBRERREPRESENTACIÓN

FUENTE: ELABORACIÓN PROPIA

TABLA 2 Dimensiones y pesos del Indicador de Ausencia de Confort

\begin{tabular}{|l|l|l|c|}
\hline \multicolumn{1}{|c|}{ DIMENSIÓN } & \multicolumn{1}{|c|}{ VARIABLE } & \multicolumn{1}{c|}{ CATEGORÍAS } & PENALIZACIÓN \\
\hline \multirow{2}{*}{$\begin{array}{l}\text { DOTACIONES CONFORT } \\
\text { (O POTENCIALES): FOMENTO DE } \\
\text { RELACIONES, AHORRO Y MAYOR } \\
\text { SEGURIDAD }\end{array}$} & Presencia de internet & No tener & 0,05 \\
\cline { 2 - 4 } & Agua caliente central & No tener & 0,2 \\
\cline { 2 - 4 } & Gas por tuberías & No tener & 0,3 \\
\hline
\end{tabular}

FUENTE: ELABORACIÓN PROPIA

Una vez creados y validados los indicadores, y tras comprobar que el análisis territorial es pertinente (el análisis chi-cuadrado señala que las diferencias no son aleatorias), se ordena la posición relativa de cada región con respecto al valor nacional. En un segundo momento, la consideración conjunta de los puntajes medios de ambos indicadores nos permite sintetizar la situación de Exclusión Residencial Ampliada (ERA) y clasificar las CC. AA. en razón de patrones de similaridad, sobre los que se realiza un análisis clúster K-medias. Por último, se establece la presencia de personas mayores que están en riesgo en cada región. Para ello se considera la distribución de la variable y se establece el umbral en el percentil 90.

\section{Resultados: cuando la desigualdad residencial se convierte en vulnerabilidad}

\section{Descriptivos territoriales y desigualdad interna}

En primer lugar, se comprueba la pertinencia de realizar el análisis mediante el contraste no paramétrico de Kruskal-Wallis. Como se muestra a continuación (Tabla 3 y Tabla 4), la dispersión de los datos para el nivel nacional es mayor en la variable ausencia de confort (Tabla 4), con una media de 0,57 y una desviación típica de 0,31 . La variable vulnerabilidad (Tabla 3) está mucho más concentrada, con una 
media de 0,15 y una desviación estándar de 0,10. La posición que ocupa la mediana muestra la presencia de valores más elevados en la falta de confort $(0,45)$ que con respecto a la vulnerabilidad (con valor 0,15 ), aunque su impacto objetivo sea mucho mayor. La distribución de la variable nos muestra que lo mayoritario es experimentar valores bajos de vulnerabilidad y ausencia de confort, lo que concuerda con la mejora generalizada en las condiciones residenciales de las personas mayores en España en los últimos treinta años (Lebrusán, 2015). También revela la existencia de personas que, incluso en este contexto de mejora, siguen sufriendo vulnerabilidad en distinto grado. Los recorridos entre la situación media y el máximo alcanzado son muy elevados, especialmente en lo que refiere a la vulnerabilidad, lo que señala la gran desigualdad interna de los territorios. Esto indica que, aun cuando la mayor parte de la población analizada presenta valores bajos de vulnerabilidad y ausencia de confort, un subgrupo de personas mayores sufre en su vivienda una combinación de carencias o problemas residenciales de gravedad. De otro modo: una región con un alto recorrido seńala que la mayor parte de las personas no sufre vulnerabilidad, pero que aquellas que la sufren, lo hacen de forma extrema.

TABLA 3 | Descriptivos sobre el índice de vulnerabilidad (ordenado según media). España, 2011

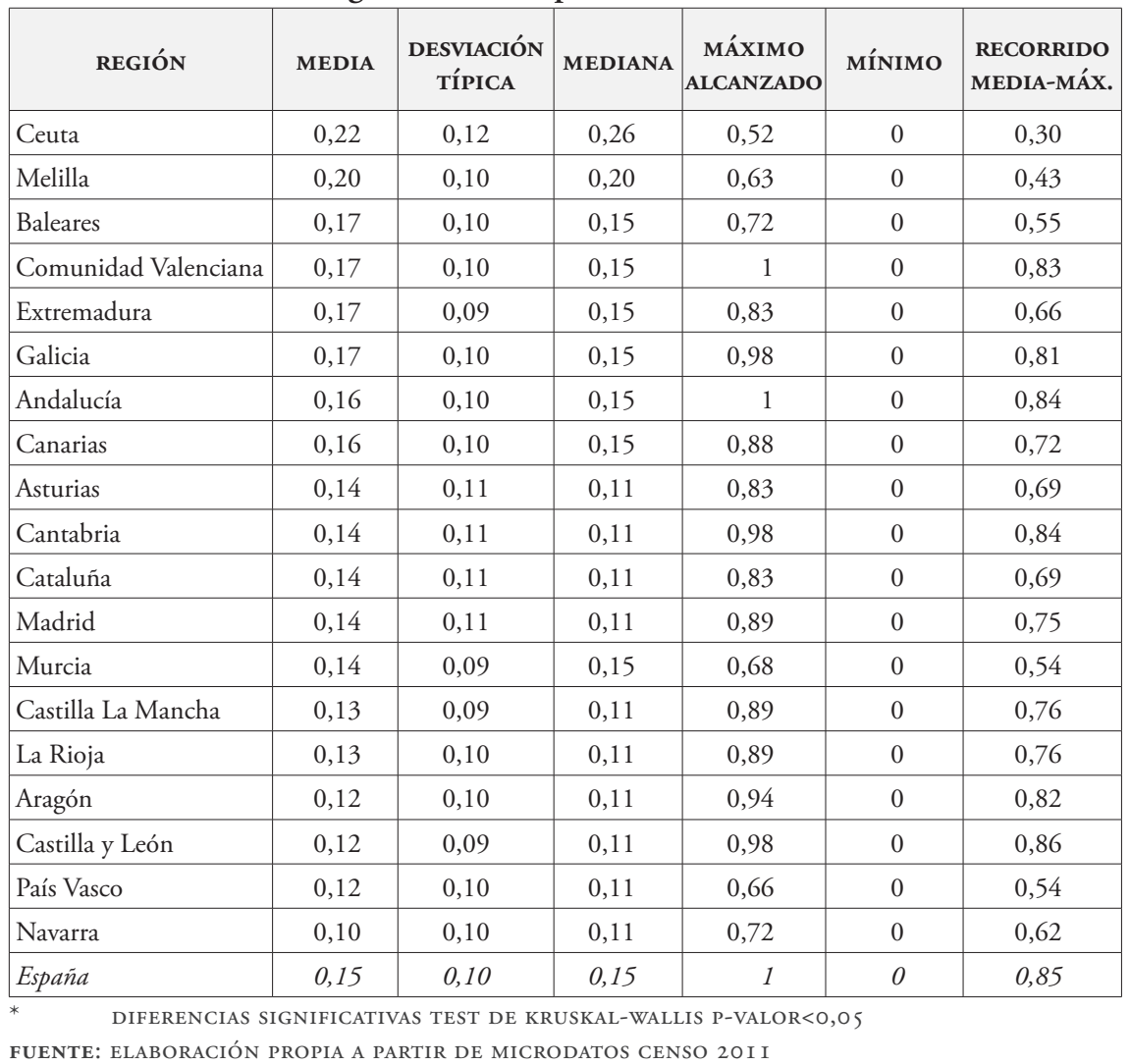


TABLa 4 Descriptivos sobre el índice de ausencia de confort (ordenado según media). España, 2011

\begin{tabular}{|l|c|c|c|c|c|c|}
\hline \multicolumn{1}{|c|}{ REGIÓN } & MEDIA & $\begin{array}{c}\text { DESVIACIÓN } \\
\text { TÍPICA }\end{array}$ & MEDIANA & $\begin{array}{c}\text { MÁXIMO } \\
\text { ALCANZADO }\end{array}$ & MÍNIMO & $\begin{array}{c}\text { RECORRIDO } \\
\text { MEDIA-MÁx. }\end{array}$ \\
\hline Ceuta & 0,88 & 0,21 & 0,91 & 1 & 0 & 0,12 \\
\hline Melilla & 0,88 & 0,21 & 1 & 1 & 0 & 0,12 \\
\hline Canarias & 0,83 & 0,26 & 0,91 & 1 & 0 & 0,17 \\
\hline Andalucía & 0,71 & 0,29 & 0,64 & 1 & 0 & 0,29 \\
\hline Extremadura & 0,71 & 0,29 & 0,64 & 1 & 0 & 0,29 \\
\hline $\begin{array}{l}\text { Comunidad Valen- } \\
\text { ciana }\end{array}$ & 0,70 & 0,30 & 0,64 & 1 & 0 & 0,3 \\
\hline Galicia & 0,70 & 0,29 & 0,64 & 1 & 0 & 0,3 \\
\hline Baleares & 0,62 & 0,29 & 0,64 & 1 & 0 & 0,38 \\
\hline Castilla La Mancha & 0,62 & 0,31 & 0,64 & 1 & 0 & 0,38 \\
\hline Murcia & 0,62 & 0,31 & 0,64 & 1 & 0 & 0,38 \\
\hline Castilla y León & 0,55 & 0,30 & 0,64 & 1 & 0 & 0,45 \\
\hline La Rioja & 0,55 & 0,30 & 0,45 & 1 & 0 & 0,45 \\
\hline Aragón & 0,54 & 0,29 & 0,55 & 1 & 0 & 0,46 \\
\hline Asturias & 0,52 & 0,31 & 0,45 & 1 & 0 & 0,48 \\
\hline Cantabria & 0,44 & 0,28 & 0,45 & 1 & 0 & 0,56 \\
\hline Cataluńa & 0,44 & 0,26 & 0,45 & 1 & 0 & 0,56 \\
\hline País Vasco & 0,44 & 0,23 & 0,45 & 1 & 0 & 0,56 \\
\hline Navarra & 0,40 & 0,29 & 0,45 & 1 & 0 & 0,6 \\
\hline Madrid & 0,38 & 0,25 & 0,45 & 1 & 0 & 0,62 \\
\hline Espańa & 0,57 & 0,31 & 0,45 & 1 & 0 & 0,43 \\
\hline$*$ & $5 I G N I F I C A T I V A S$ & TEST DE KRUSKAL-WALLIS P-VALOR<0,05 & \\
\hline
\end{tabular}

FUENTE: ELABORACIÓN PROPIA A PARTIR DE MICRODATOS CENSO 20 I I

Los descriptivos previos señalan varias cosas: i) clara desigualdad entre las regiones en ambos indicadores; ii) existencia de desigualdad interna en todos los territorios, aunque mucho más significativa en unas regiones que en otras (columna recorrido media-máximo); iii) mayor diferencia entre las medias en el índice falta de confort -recorrido de 0,50, entre el mínimo en Madrid $(0,38)$ y el máximo en Ceuta $(0,88)$ - que en el de vulnerabilidad: de 0,12 puntos entre Navarra $(0,10)$ y Ceuta $(0,22)$; iv) cierta congruencia territorial en el ordenamiento relativo de ambos indicadores. Encontramos las excepciones de Castilla y León, Castilla La Mancha y Canarias, que experimentan clara peor posición relativa en el indicador ausencia de confort que en el de vulnerabilidad. Podría haberse esperado un peor posicionamiento comparativo respecto al índice de ausencia de confort en las regiones que tienen un mayor peso rural -debido a los componentes del propio índice-, pero a excepción de las referidas, las variaciones en el orden no son muy grandes.

Navarra, seguida de País Vasco, Castilla y León y Aragón son las comunidades que presentan un menor índice medio de vulnerabilidad, dejando las peor posicionadas fuera de la península (Ceuta y Melilla con niveles mucho más elevados que el resto de regiones, seguidas por Baleares). Dentro de la península, y con un 
nivel muy similar al de Baleares, tendríamos Galicia, Extremadura y la Comunidad Valenciana. Por encima de la vulnerabilidad media nacional $(0,15)$ también quedan Canarias y Andalucía. Si analizamos los descriptivos de este indicador, vemos, sin embargo, que el máximo (1) se alcanza únicamente en Andalucía y la Comunidad Valenciana, que serían así las regiones que registran una mayor desigualdad interna.

El indicador de ausencia de confort es menos favorable entre las personas de la Comunidad de Madrid, que registra la mejor posición (valor más bajo para la ausencia de confort), seguida de Navarra, Cantabria, Cataluña y País Vasco. Las regiones con mayor ausencia de confort medio de nuevo quedan fuera de la península: Melilla y Ceuta seguidas por Canarias. Con un nivel no tan negativo, pero con peores valores que la media nacional $(0,57)$, destacan Andalucía y Extremadura, con niveles similares, seguidas por Galicia y la Comunidad Valenciana (ambas con 0,70 ) y, a continuación, Murcia, Castilla La Mancha y Baleares (con 0,62). En este caso el valor máximo (1) se alcanza en todas las regiones.

A fin de simplificar la complejidad de los dos indicadores y superar su análisis descriptivo, se propone la creación de un clúster para analizar qué regiones muestran resultados similares y poder determinar qué grupo de comunidades necesita mayor atención.

\section{¿Existe un modelo territorial? La Exclusión Residencial Ampliada (ERA)}

El análisis anterior apunta a que existe una clara desigualdad en la situación residencial de las personas mayores en Espańa, en la que el factor territorial tiene un papel importante. Como se indicó, la medición de la ausencia de confort por sí sola pudiera no resultar especialmente ilustrativa de las inadecuaciones residenciales, pero ella sí ańade nuevos riesgos dentro del hogar, especialmente cuando se producen situaciones de vulnerabilidad. Además, se constata estadísticamente que ambas variables están relacionadas. Considerando ambos indicadores, podremos hablar de situaciones de Exclusión Residencial Ampliada.

Tras observar los diferentes puntajes y dado que las delimitaciones político-administrativas regionales refieren distinta efectividad en la resolución de los problemas residenciales, se propone una clasificación de las regiones mediante un clúster de cuatro grupos, cuya caracterización puede comprobarse en la Figura 1. Esta técnica estadística clasifica los territorios según el criterio de similaridad proporcionado por los centroides (medias generales o centros de ambas variables), ordenados de peor posición (ERA grado I o superior) a mejor posición (ERA grado IV o media baja).

El primer grupo clúster sería el más desfavorecido de todos, pues alcanza los valores superiores en ambos índices (ERA I o de grado superior). El segundo grupo (ERA grado II o elevado) estaría conformado por los territorios que ocupan el siguiente valor con respecto a ambos indicadores. Por último, los grupos tercero y cuarto (ERA grado III y ERA grado IV, media alta y media baja, respectivamente) presentan mejores valores. Los criterios de adjudicación a uno u otro grupo responden a la conjugación de las puntuaciones de ambas variables (menor vulnerabilidad/mayor ausencia de confort o viceversa). Puesto que las distancias en confort son más elevadas, se ha considerado primar esta diferencia. Esto significa que el grupo III tiene una puntuación ligeramente más baja con respecto a la vulnerabilidad que 
el grupo IV, pero muy superior con respecto a la ausencia de confort. La Figura 2 expresa, siguiendo la clasificación anterior, el modelo regional de ERA.

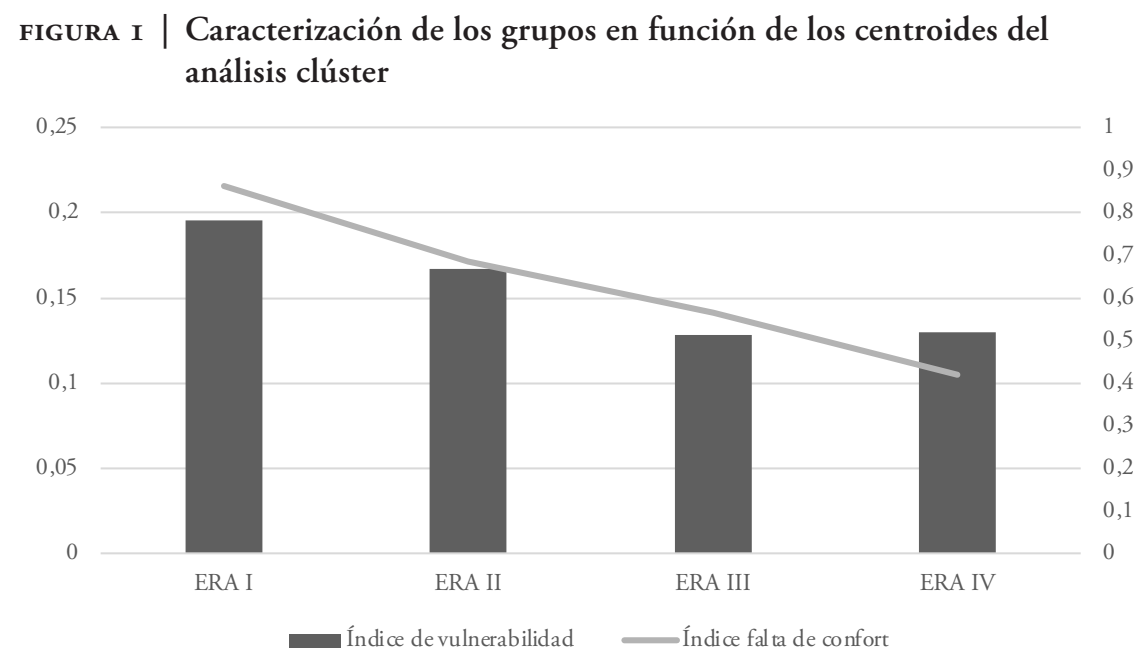

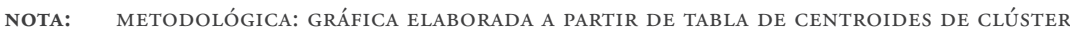
FUENTE: ELABORACIÓN PROPIA A PARTIR DE MICRODATOS CENSO 2 OI I

El mapa (Figura 2) muestra una gradación del impacto de la Exclusión Residencial Ampliada, que se sintetiza de la siguiente manera:

- ERA grado I: Las delimitaciones político-administrativas que presentan una situación más desfavorable son las ciudades autónomas de Melilla y Ceuta junto a Canarias.

- ERA grado ir: En mejor situación, pero registrando las peores posiciones dentro de la península, se encontrarían Galicia, Comunidad Valenciana, Andalucía, Extremadura -siendo la primera comunidad la más representativa del grupo-. Fuera de la península, con valores lo suficientemente negativos para ser parte del ERA II, encontramos Baleares.

El resto de CC. AA. se encuentra en mejor situación con respecto a ambos indicadores, con diferencias según si el criterio de optimización responde a la ausencia de vulnerabilidad o a la no ausencia de confort.

- ERA grado III: Este grupo, por orden de representatividad (mayor a menor) está conformado por La Rioja, Castilla y León, Aragón, Asturias, Murcia y Castilla La Mancha.

- $\quad$ ERA grado IV: Por último, el grupo ERA IV combina un ligero empeoramiento en la vulnerabilidad respecto al grupo ERA III, pero presenta una mejora considerable en confort. Este grupo es el que se encuentra en mejor situación dentro de España, e incluye las comunidades (de más representativa del clúster a menos) de Cantabria, País Vasco, Cataluña, Navarra y Madrid. 
FIgURA 2 | Modelo regional de la Exclusión Residencial Ampliada (ERA) para los mayores de 65 ańos. España, 2011

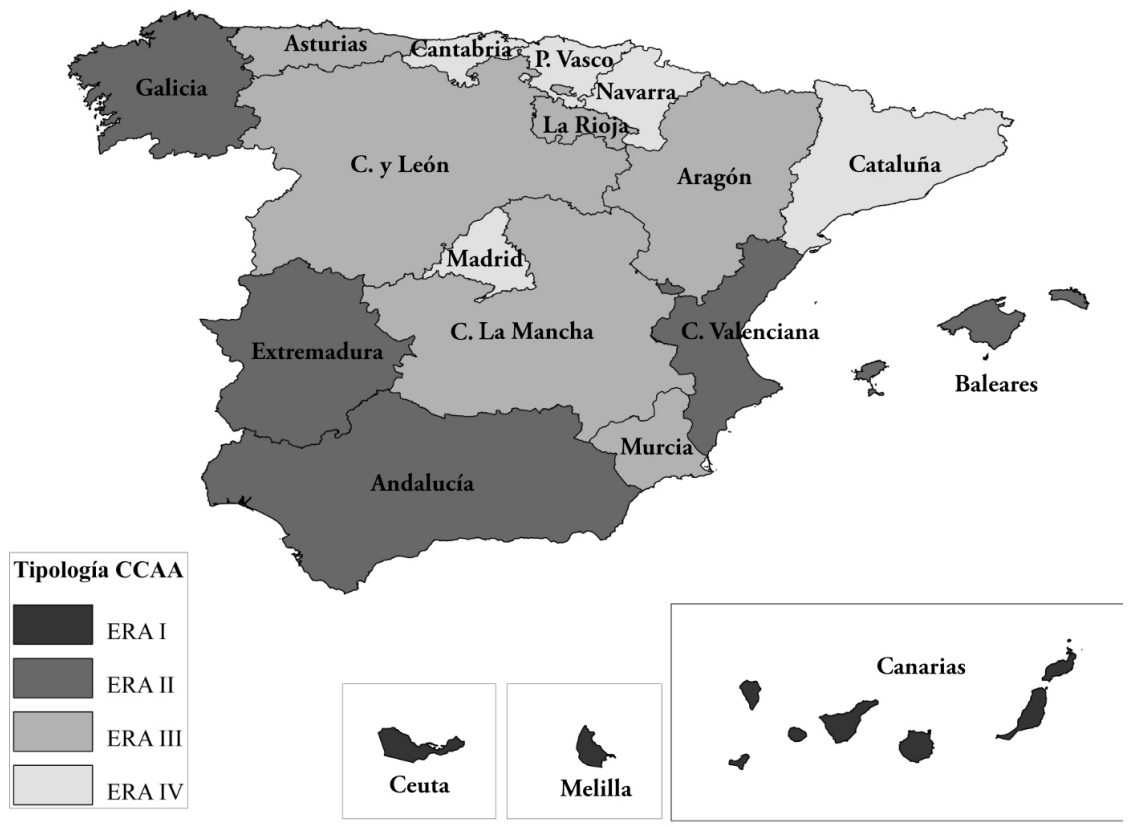

FUENTE: ELABORACIÓN PROPIA A PARTIR DE MICRODATOS CENSO 20 I I

Estos resultados permiten constatar una clara desigualdad residencial en el mapa espańol, presentando mayor malestar las personas mayores residentes en regiones que quedan fuera de la península, además de Extremadura, Andalucía, Galicia y Comunidad Valenciana.

Queda demostrada la desigualdad residencial respecto a ambos indicadores y respecto al indicador de Exclusión Residencial Ampliada. A continuación, y si bien se ha apuntado la existencia de distancia entre medias y valores máximos (también de recorrido desigual entre unas regiones y otras), necesitamos conocer en qué regiones las situaciones de vulnerabilidad extrema tienen un impacto mayor. Debido a la configuración del indicador, serán estas las regiones donde las intervenciones públicas dirigidas a luchar contra la exclusión social y residencial serán más urgentes.

\section{Personas mayores en situación de riesgo extremo: desigualdades inter e intraterritoriales}

Consideramos en situación de riesgo extremo a las personas que, teniendo en cuenta el comportamiento de la variable de vulnerabilidad residencial, se encuentran en el umbral superior. El punto a partir del cual la situación se ha establecido como intolerable se determina con el percentil 90, que en vulnerabilidad corresponde al valor 0,2615. El valor, con recorrido 0-1, pudiera parecer no muy elevado. Sin embargo, el contenido del indicador refiere características de extrema gravedad, que quedan 
por debajo de los parámetros mínimos de habitabilidad. Estamos, por lo tanto, ante situaciones de vivienda infraestándar. Estas personas no habrían conseguido dar respuesta a las necesidades residenciales más básicas y estarían experimentando una acumulación de problemas que dificulta su continuidad en la vivienda y que afecta muy negativamente su calidad de vida.

Esta nueva variable categórica que mide la condición de estar en riesgo o no señala que hay 1.596 .675 personas mayores de 65 años en situación de vulnerabilidad extrema. En otras palabras: el 20,1\% de las personas mayores en España está en situación de riesgo desde el punto de vista residencial.

La cuestión ahora es hallar cómo se reparten las situaciones extremas en el territorio español y si existe desigualdad interna en las regiones. Esta vendría explicada por bajos valores medios pero elevada presencia de población en situación residencial extremadamente negativa. Para ello, y a partir de la tabla de contingencia, se crea el mapa de reparto de la vulnerabilidad extrema (Figura 3).

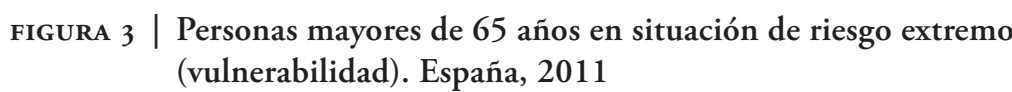

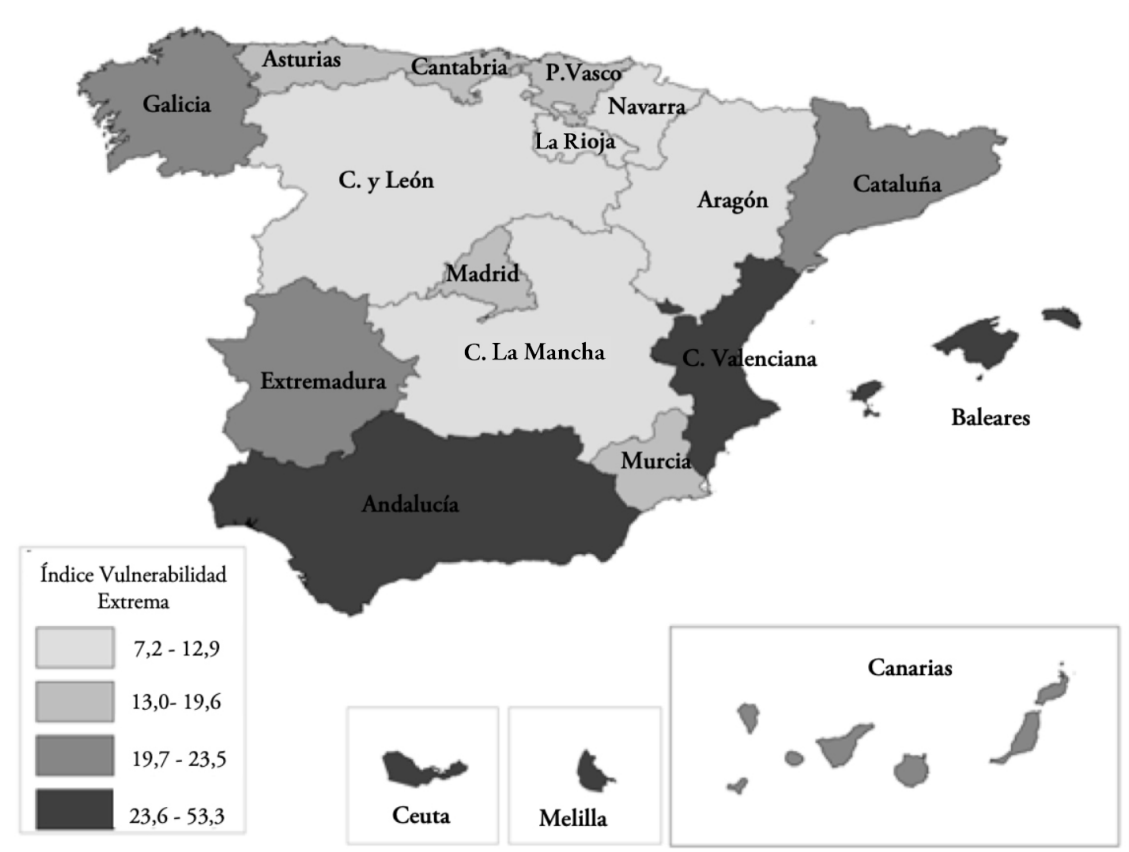

NOTA: CHI-CUADRADO DE PEARSON $=$ I 83876,8 P-VALOR $=0,000$

FUENTE: ELABORACIÓN PROPIA A PARTIR DE MICRODATOS CENSO 20 I I

La gran desigualdad del mapa español se mueve en un rango de más de 46 puntos porcentuales. Encabezando el ranking, el 53,3\% y el 42,6\% de los mayores residentes en Ceuta y Melilla, respectivamente, sufren situaciones de vulnerabilidad extrema. A las ciudades autónomas les siguen la Comunidad Valenciana y las 
Baleares, antes que Andalucía, a diferencia de lo que sucedía con la vulnerabilidad media. El siguiente clúster (entre 23,5\% y 19,7\% de mayores que registran situaciones extremas) está conformado por Galicia, Cataluña (estas dos por encima del valor nacional) y Extremadura. La meseta central, a la que se suman Aragón, algunas regiones del norte y Murcia, quedan mejor posicionadas. Navarra presenta el porcentaje menor de personas en situación de vulnerabilidad extrema (7,2\%).

\section{Discusión de resultados: intervenciones urgentes e intervenciones de apoyo}

El análisis de los diferentes indicadores confirma que en Espańa hay un número elevado de personas mayores que no tienen adecuadamente cubiertas sus necesidades residenciales. Más aún, se producen situaciones de riesgo residencial que estarían impidiendo su adecuada integración y participación en la sociedad. Las condiciones residenciales no solo tienen un efecto determinante en la calidad de vida de la persona mayor, sino que son también clave en su independencia y, por tanto, en el retraso de la necesidad de cuidado. Cabe añadir que las condiciones de la vivienda tendrán un efecto decisivo tanto en la capacidad de recibir cuidados asociados a la dependencia como a su calidad (Redondo et al., 2015). Estas son cuestiones que necesitan de atención urgente, especialmente en un país que ya cuenta con una proporción de adultos mayores muy superior a la de algunos países iberoamericanos (Monteverde et al., 2016) y donde la pirámide actual señala una tendencia a su aumento.

El malestar extremo detectado es sensible al factor territorial, lo que señala un reparto desigual en el mapa espańol, tanto a nivel interterritorial como intraterritorial. Los resultados se sintetizan en el siguiente ranking ordenado (Tabla 5), que permite establecer si los niveles medios y extremos están relacionados o si bien las regiones presentan gran desigualdad interna (vulnerabilidad media baja pero elevada presencia de situaciones extremas), a la vez que observamos su posicionamiento relativo respecto a Espańa.

TABLA 5 | Ranking ordenado respecto a porcentaje de personas en situación de vulnerabilidad extrema, datos poblacionales y valor de vulnerabilidad media, por regiones. España, 2011

\begin{tabular}{|c|c|c|c|c|c|c|c|c|}
\hline \multicolumn{4}{|c|}{ MAYORES EN VULNERABILIDAD EXTREMA } & \multicolumn{2}{|c|}{$\begin{array}{c}\text { DATOS } \\
\text { POBLACIONALES } \\
\end{array}$} & \multicolumn{3}{|c|}{$\begin{array}{l}\text { VALOR VULNERABILIDAD } \\
\text { MEDIA }\end{array}$} \\
\hline ORDEN & $\begin{array}{l}\text { COMUNIDADES } \\
\text { AUTÓNOMAS }\end{array}$ & $\%$ & $\begin{array}{c}\text { MAYORES } \\
\text { EN } \\
\text { VULNERA- } \\
\text { BILIDAD }\end{array}$ & \begin{tabular}{|c|}
$\% 65+$ \\
SOBRE \\
TOTAL \\
POBL. EN \\
VIVIENDAS
\end{tabular} & $\begin{array}{c}65+\text { EN } \\
\text { VIVIEN- } \\
\text { DAS }\end{array}$ & ORDEN & $\begin{array}{l}\text { COMUNIDADES } \\
\text { AUTÓNOMAS }\end{array}$ & VALOR \\
\hline 1 & Ceuta & 53,3 & 4.832 & 10,9 & 9.065 & 1 & Ceuta & 0,22 \\
\hline 2 & Melilla & 42,6 & 3.353 & 9,8 & 7.870 & 2 & Melilla & 0,20 \\
\hline 3 & $\begin{array}{l}\text { Com. Valen- } \\
\text { ciana }\end{array}$ & 29,6 & 249.892 & 16,9 & 844.230 & 3 & Baleares & 0,17 \\
\hline 4 & Baleares & 28,1 & 43.391 & 14,1 & 154.415 & 4 & $\begin{array}{l}\text { Com. } \\
\text { Valenciana }\end{array}$ & 0,17 \\
\hline 5 & Andalucia & 25,5 & 321.563 & 15,1 & 1.261 .030 & 5 & Extremadura & 0,17 \\
\hline
\end{tabular}




\begin{tabular}{|c|c|c|c|c|c|c|c|c|}
\hline \multicolumn{4}{|c|}{ MAYORES EN VULNERABILIDAD EXTREMA } & \multicolumn{2}{|c|}{$\begin{array}{c}\text { DATOS } \\
\text { POBLACIONALES }\end{array}$} & \multicolumn{3}{|c|}{$\begin{array}{l}\text { VALOR VULNERABILIDAD } \\
\text { MEDIA }\end{array}$} \\
\hline ORDEN & $\begin{array}{c}\text { COMUNIDADES } \\
\text { AUTÓNOMAS }\end{array}$ & $\%$ & $\begin{array}{l}\text { MAYORES } \\
\text { EN } \\
\text { VULNERA- } \\
\text { BILIDAD }\end{array}$ & $\begin{array}{c}\% 65+ \\
\text { SOBRE } \\
\text { TOTAL } \\
\text { POBL. EN } \\
\text { VIVIENDAS }\end{array}$ & $\begin{array}{c}65+\text { EN } \\
\text { VIVIEN- } \\
\text { DAS }\end{array}$ & ORDEN & $\begin{array}{l}\text { COMUNIDADES } \\
\text { AUTÓNOMAS }\end{array}$ & VALOR \\
\hline 6 & Galicia & 23,5 & 146.104 & 22,5 & 621.720 & 6 & Galicia & 0,17 \\
\hline 7 & Canarias & 20,7 & 59.259 & 13,8 & 286.275 & 7 & Andalucía & 0,16 \\
\hline 8 & Cataluña & 20,4 & 254.051 & 16,7 & 1.245 .350 & 8 & Canarias & 0,16 \\
\hline 9 & Extremadura & 19,7 & 40.839 & 18,9 & 207.305 & 9 & Asturias & 0,14 \\
\hline 10 & Asturias & 19,6 & 46.740 & 22,3 & 238.470 & 10 & Cantabria & 0,14 \\
\hline 11 & Murcia & 18,8 & 38.677 & 14,1 & 205.730 & 11 & Cataluña & 0,14 \\
\hline 12 & Cantabria & 18,0 & 19.755 & 18,6 & 109.750 & 12 & Madrid & 0,14 \\
\hline 13 & Madrid & 17,4 & 167.609 & 15,1 & 963.270 & 13 & Murcia & 0,14 \\
\hline 14 & País Vasco & 13,9 & 59.012 & 19,5 & 424.550 & 14 & $\begin{array}{l}\text { Castilla La } \\
\text { Mancha }\end{array}$ & 0,13 \\
\hline 15 & La Rioja & 12,9 & 7.567 & 18,4 & 58.660 & 15 & La Rioja & 0,13 \\
\hline 16 & Aragón & 11,8 & 30.681 & 19,5 & 260.005 & 16 & Aragón & 0,12 \\
\hline 17 & $\begin{array}{l}\text { Castilla La } \\
\text { Mancha }\end{array}$ & 10,7 & 38.576 & 17,2 & 360.520 & 17 & Castilla y León & 0,12 \\
\hline 18 & Castilla y León & 10,1 & 57.072 & 22,5 & 565.065 & 18 & País Vasco & 0,12 \\
\hline 19 & Navarra & 7,2 & 7.956 & 17,4 & 110.500 & 19 & Navarra & 0,10 \\
\hline \multicolumn{2}{|l|}{ Espańa } & 20,1 & 1.594 .689 & 17,0 & 7.933 .775 & \multicolumn{2}{|l|}{ Espańa } & 0,15 \\
\hline
\end{tabular}

FUENTE: ELABORACIÓN PROPIA A PARTIR DE MICRODATOS CENSO 2 OI I

El análisis territorial resulta complejo y sujeto a matices: las ciudades autónomas de Ceuta y Melilla mantienen su posición al frente de la vulnerabilidad; no solo el nivel medio de vulnerabilidad es más elevado que en el resto del territorio espańol, sino que también hay un mayor porcentaje de mayores en situación de riesgo extremo. A pesar de ser regiones jóvenes, con un porcentaje muy bajo de personas mayores de 65 ańos, en torno a la mitad de estas personas se encuentra en una situación intolerable.

Baleares y la Comunidad Valenciana intercambian sus posiciones en el ordenamiento relativo, pero ambas muestran valores de vulnerabilidad preocupantes, comparativamente muy superiores al del conjunto nacional: casi un tercio de los mayores está en situación de vulnerabilidad extrema. La situación de la Comunidad Valenciana sorprende especialmente, pues cabría esperar que una región con un elevado Producto Interior Bruto (РІВ) ${ }^{5}$ y que alberga una de las mayores ciudades en Espańa (Valencia), hubiera dispuesto de mayores recursos para luchar contra la infravivienda. Les siguen Andalucía, Canarias y Cataluña, todas con mayor porcentaje de mayores en vulnerabilidad extrema que la media nacional; y Cataluña, además, con una gran desigualdad extrema. Estas serían las regiones que necesitan medidas más urgentes, y que acusan una mayor incidencia de la vulnerabilidad

5 Según la Contabilidad Regional de España, en 2019 el piв de la Comunidad Valenciana alcanzó el cuarto lugar en el país, con 115.456 millones de euros autónomos (INE, 2019a). 
extrema. En conjunto, albergan casi el $67 \%$ de la población mayor total que se encuentra en situación de riesgo extremo. Como señalábamos en la introducción, y siguiendo la estructura de análisis de Lawton y Nahemow (1973), al producirse una demanda excesiva por parte del entorno (en este caso, las malas condiciones de la vivienda) el equilibrio con las capacidades de la persona no será posible, lo que impediría finalmente la independencia, incluso si la vejez no viene acompañada de pérdida de competencia significativa

El resto de las regiones se encuentra por debajo del promedio nacional, lo que no implica ausencia de vulnerabilidad. Cabe señalar el caso de Madrid: si bien queda bajo el puntaje medio nacional, su elevada población hace que albergue casi el $11 \%$ de la población mayor en situación de vulnerabilidad extrema. Navarra presenta la mejor de las situaciones con respecto a ambas mediciones, y sería el ejemplo que debiera seguir el resto de comunidades; lo mismo ocurre en el caso de Castilla y León, a pesar de registrar un peso elevado de población mayor en comparación con otras comunidades. La Rioja y Aragón también se encuentran en buenas situaciones con respecto a ambas mediciones (la media y la extrema) y no cambian su posición en el ranking, lo que indica menor desigualdad intraterritorial. Puesto que la seguridad y el bienestar dentro de las viviendas puede ser un factor de protección de la salud al disminuir accidentes, reducir el estrés y mejorar la calidad de vida (Garay et al., 2016), podemos asumir que estas regiones estarían planteando formas de protección contra la dependencia y la mala calidad de vida en la vejez. En este sentido, una buena calidad de la vivienda podría incluso asociarse a estrategias preventivas en materia de salud en la vejez y, por lo tanto, conducir a una menor necesidad de inversión en cuidados paliativos. Esta, no obstante, es una hipótesis que abre una nueva línea de investigación territorial.

Las diferencias apuntan que los sistemas de vivienda regionales -entendiendo como tal aquellos sistemas que ponen en relación hogares, políticas y comportamientos residenciales, así como necesidades no resueltas (Módenes \& López-Colás, 2014) - presentan diversos grados de efectividad, confirmando lo que se ha denominado como heterogeneidad homogénea (Echaves, 2017). En este sentido, y en lo que refiere a la posible desigualdad residencial, es necesario señalar que la competencia en materia de vivienda corresponde a cada comunidad autónoma, "facultada para desarrollar su política de vivienda con cargo a sus propios recursos" (Echaves, 2016, p. 28). Los resultados muestran la necesidad de implementar acciones residenciales regionales con distinto grado de urgencia para reducir estas situaciones de extrema desigualdad. Comprobamos, además, que el peso relativo de la población mayor de 65 años no es indicativo de una mejor o peor resolución de las necesidades residenciales. Por último, la existencia de desigualdad interna en cada territorio plantea nuevos desafíos en el diseño e implementación de las políticas regionales y locales dirigidas a la detección de las necesidades residenciales de las personas mayores, y de la respuesta a ellas. 


\section{Conclusiones, limitaciones y líneas futuras de trabajo}

No solo se produce una gran desigualdad en términos de calidad de vivienda, sino que numerosos mayores residen en situaciones de infravivienda en el territorio español. La variable territorial resulta clave en la distribución de este malestar: aunque en todas las regiones se detectan problemáticas de gravedad, el malestar residencial es claramente más acusado en unas zonas que en otras. Si bien la mayoría de los adultos mayores no sufre problemas extremos, la aplicación de los indicadores y su contenido permiten concluir: i) que existen grupos de población que permanecen en sus hogares en una muy mala situación, impidiendo así una vejez de calidad; y ii) las carencias y problemas que definen las situaciones de malestar residencial, especialmente en lo que refiere a la variable vulnerabilidad, son de extrema gravedad. Es esperable, además, que estas características residenciales dificulten la provisión de cuidados en la propia vivienda, pudiendo incluso acelerar la necesidad y cantidad de los mismos al funcionar la mala condición de la vivienda como un impedimento en la autosuficiencia y la independencia. Espańa corre el riesgo de que el aumento de la esperanza de vida (actualmente una de las más elevadas del mundo) no sea equivalente a una buena vejez.

Este malestar residencial, que afecta a un número elevado de personas mayores, pone en duda la efectividad del sistema residencial: en la etapa final de su ciclo vital estas personas no han logrado solventar sus necesidades residenciales, lo que apuntaría a la ineficacia del sistema residencial, que no permite que todos los hogares den respuesta de manera particular a sus propias necesidades. A la vista de los datos, puede afirmarse que tampoco se han aportado soluciones suficientes desde el sistema público. Si bien entre las causas de la vulnerabilidad residencial se esperarían múltiples motivos imbricados entre sí, situación en que el funcionamiento del mercado de vivienda y las propias políticas puedan ocupar un lugar más o menos preponderante, lo cierto es que ni las políticas públicas regionales ni las nacionales están dando respuesta adecuada a situaciones de vulnerabilidad.

El análisis territorial complementa estas conclusiones: si bien España a nivel nacional no ha sido capaz de solventar problemas de gravedad, la desigualdad regional existente expresa diferencias atribuibles a las administraciones territoriales. Es decir, la desigualdad territorial indica que algunas administraciones han sido más ineficaces en la implementación de políticas de vivienda y en la creación de condiciones óptimas para que los hogares den respuesta a las necesidades de las personas mayores. Tampoco hay una respuesta paliativa ante estas situaciones de riesgo en la vejez.

El diagnóstico apunta a la necesidad de una respuesta con mayor urgencia y contundencia en las regiones y las ciudades autónomas que quedan fuera de la península, pero también en la Comunidad Valenciana y Andalucía. No solo los puntajes de vulnerabilidad media son más elevados y registran una mayor Exclusión Residencial Ampliada, sino que la presencia de personas que sufren situaciones extremas es mayor. Su situación señala una gran desigualdad en el territorio nacional, con variaciones extremas. Esto evidencia lo acertado de la recomendación del comité DESC que referíamos en la introducción: en España se produce la 
vulneración del derecho a una vivienda adecuada y se constata una clara disparidad entre comunidades autónomas, en ausencia de coordinación con el gobierno central en materia de bienestar social. A pesar de existir una política nacional, son las administraciones autonómicas las que finalmente influyen en el grado de bienestar residencial, acusando diferentes niveles de capacidad (o incapacidad) para resolver las necesidades habitacionales de su población. Es, por lo tanto, necesario reclamar una mejor coordinación entre las políticas nacionales y una mayor proactividad por parte de las regionales en materia de vivienda e integración social dirigida a las personas mayores. Cabe preguntarse si, ante el esperable incremento de la población mayor en Espańa, aumentará la existencia de vulnerabilidad residencial extrema.

El estudio presenta limitaciones. La primera de ellas es la que se asocia a la fuente de la que proceden los datos y su antigüedad. Si bien la investigación realiza un ejercicio necesario al crear índices sintéticos y validados que permiten detectar las problemáticas más graves en el mapa español, sería necesario contemplar un mayor número de variables que, sin ser estrictamente residenciales, contribuyen de manera evidente al malestar residencial de la población mayor. Por otra parte, este diagnóstico es imprescindible para señalar la urgencia territorial de las necesidades (en referencia a aquellas regiones sobre las que deben dirigirse de manera prioritaria acciones contra el malestar residencial), pero sería necesario ampliarlo incluyendo otro tipo de variables, así como la identificación de "buenas prácticas" en materia de vivienda. También será necesario analizar las situaciones residenciales de la población que entrará en la vejez en los próximos ańos y, como se ha seńalado, las diversas implicaciones que ello tiene en materia de cuidados. Por último, detectar la evolución de las carencias residenciales serviría también para conocer el impacto de las políticas de vivienda en el periodo 2011-2021. Para eso aún tendremos que esperar un poco. Todas estas son futuras líneas de investigación con las que complementar la información que aquí se expone para luchar contra la infravivienda en la vejez y permitir que el envejecimiento en la vivienda (aging in place) se acompańe de una buena calidad de vida.

\section{Referencias bibliográficas}

Alberich, J. (2017). Nuevas metodologías para el estudio de la movilidad habitual: el uso de los registros administrativos en la movilidad por motivos de estudio en Catalunya. Scripta Nova. Revista Electrónica de Geografía y Ciencias Sociales, 21. https://doi.org/10.1344/ sn2017.21.18343

Antón, F., Cortés, L., Martínez, C. \& Navarrete, J. (2008). La exclusión residencial en España. En V. Renes (Coord.), VI Informe sobre exclusión social y desarrollo social en España. Cáritas-Fundación foessa (Fomento de Estudios Sociales y Sociología Aplicada).

Ayala, L. \& Navarro, C. (2004). Multidimensional indices of housing deprivation with application to Spain. Papeles de trabajo del Instituto de Estudios Fiscales. Serie Economía, $\mathrm{n}^{\circ}$ 12. https://www.ief.es/docs/destacados/publicaciones/papeles_trabajo/2004_12.pdf 
Barratt, J. (2007). International perspectives on aging and disasters. Generations, 31(4), 57-60. https://www.jstor.org/stable/26555566

Benito, J. C. (2019). Los pronunciamientos del comité DesC sobre Derecho a la Vivienda relativos a Espańa. Respuestas jurisprudenciales y legislativas. Lex Social: Revista de Derechos Sociales, 9(2), 579-603. https://doi.org/10.46661/lexsocial.4228

Caradec, V. (2012). Sociologie de la vieillesse et du vieillissement. Armand Colin.

Cortés, L. (2004). Indagaciones sobre la exclusión residencial. Arxius de Ciències Socials, 10, $39-55$.

Costa-Font, J., Elvira, D. \& Mascarilla-Miró, O. (2009). 'Ageing in place'? Exploring elderly people's housing preferences in Spain. Urban Studies, 46(2), 295-316. https://doi. org/10.1177/0042098008099356

Delgado, M. (Coord.), Zamora, F., Barrios, L. \& Cámara, N. (2012). Maternidad adolescente $y$ anticoncepción en las comunidades autónomas españolas. Consejo Superior de Investigaciones Científicas (csic), España.

Echaves, A. (2016). Juventud, emancipación residencial y sistema de provisión de vivienda: las divergencias autonómicas del modelo español (Tesis doctoral inédita). Universidad Complutense de Madrid, Madrid, España. https://eprints.ucm.es/id/eprint/35557

Echaves, A. (2017). Emancipación residencial y sistema de provisión de vivienda: la heterogeneidad autonómica del modelo español. REIS: Revista Española de Investigaciones Sociológicas, 51-71. https://doi.org/10.5477/cis/reis.159.51

Eurostat. (2019). European Union Statistics on Income and Living Conditions; (ilc_mdho07). Serie 2011-2018. [Conjunto de datos]. Eurostat. https://ec.europa.eu/eurostat/data/ database

Faura-Martínez, Ú., Lafuente-Lechuga, M. \& García-Luque, O. (2016). Riesgo de pobreza o exclusión social: evolución durante la crisis y perspectiva territorial. REIS: Revista Española de Investigaciones Sociológicas, (156), 59-76. https://doi.org/10.5477/cis/ reis. 156.59

Fernández-Carro, C. (2013). 'Ageing in Place' in Europe: a multidimensional approach to independent living in later life (Tesis doctoral inédita). Universitat Autónoma de Barcelona, Barcelona, España. http://hdl.handle.net/10803/129081

Fernández-Carro, C. \& Evandrou, M. (2014). Staying put: factors associated with ageing in one's 'lifetime home'. Insights from the European Context. Research on Ageing and Social Policy, 2(1), 28-56. http://dx.doi.org/10.4471/rasp.2014.02

Fernández-García, M., Moreno-Márquez, G., Iglesias-Martínez, J. \& Oleaga-Páramo, J. A. (2018). El impacto de la crisis sobre la inmigración ecuatoriana en España. Convergencia, 25(76), 169-190. https://doi.org/10.29101/crcs.v25i76.4391

Fernández-Mayoralas, G. F. \& Rojo Pérez, F. (2005). Análisis de la vivienda familiar y sus ocupantes en el distrito centro de Madrid. Anales de Geografia de la Universidad Complutense, 25. https://revistas.ucm.es/index.php/AGUC/article/view/AGUC05051 $10281 \mathrm{~A}$

Forsyth, A. \& Molinsky, J. (2020). What is aging in place? Confusions and contradictions. Housing Policy Debate, 31(2), 181-196. https://doi.org/10.1080/10511482.2020.17 93795

Frank, J. (2001). How long can I stay? The dilemma of aging in place in assisted living. Journal of Housing for the Elderly, 15(1-2), 5-30. https://doi.org/10.1300/J081v15n01_02 
Garay, S., Montes de Oca, V. \& Hebrero, M. (2016). Los entornos y el envejecimiento en Iberoamérica: análisis a partir de las condiciones de la vivienda. Notas de Población, 42(101), 109-125. https://doi.org/10.18356/6af188c9-es

Gubrium, J. F. (1973). The myth of the golden years: A socio-environmental theory of aging. Charles C. Thomas.

Hernández, R., Fernández, C. \& Baptista, P. (2014). Metodología de la investigación. McGrawHill.

Instituto Nacional de Estadística (INE), España. (2011). Metodología de cálculo de las cifras de población censal. https://www.ine.es/censos2011/censos2011_meto_calculo.pdf

Instituto Nacional de Estadística (INE), España. (2019a). Contabilidad Regional de España. Resultados. [Conjunto de datos]. https://www.ine.es/dyngs/INEbase/es/operacion.ht m?c=Estadistica_C\&cid=1254736167628\&menu=resultados\&idp $=1254735576581$ \#!tabs-1254736158133

Instituto Nacional de Estadística (INE), España. (2019b). Encuesta de Condiciones de Vida. [Conjunto de datos]. https://www.ine.es/dyngs/INEbase/es/operacion.htm?c=Estadist ica_C\&cid $=1254736176807 \&$ menu $=u l t i D a t o s \& i d p=1254735976608$

Jurado, A. \& Pérez, J. (2007). La dimensión territorial en la investigación sobre pobreza y privación. En F. Vidal \& V. Renes (Coords.), La agenda de investigación en exclusión y desarrollo social (pp. 165-178). Cáritas Española.

Jurado, A. \& Pérez, J. (2010). Dimensión territorial de la pobreza en España. Revista Española del Tercer Sector, 15, 43-66. https://dialnet.unirioja.es/servlet/articulo?codigo=3289672

Jurado, A. \& Pérez, J. (2014). Disparidades entre las comunidades autónomas españolas en el periodo 2007-2012. Documento de trabajo 2.9, Fundación FOESSA. http://www. foessa2014.es/informe/uploaded/documentos_trabajo/15102014141722_6189.pdf

Lawton, M. P. (1977). An ecological theory of aging applied to elderly housing. Journal of Aging and Environment, 31(1), 8-10. https://doi.org/10.1080/10464883.1977.11102585

Lawton, M. P. (1983). Environment and other determinants of weil-being in older people. The Gerontologist, 23(4), 349-357. https://doi.org/10.1093/geront/23.4.349

Lawton, M. P. \& Nahemow, L. (1973). Ecology and the ageing process. En C. Eisdorpher \& M. P. Lawton (Eds.), Psychology and Adult Development and Aging (pp. 619-674). American Psychological Association.

Lebrusán, I. (2015). La inadecuación residencial después de los 65 años: carencias en viviendas que no se adaptan. Documentación social, (176), 37-54.

Lebrusán, I. (2017). La vivienda en la vejez; problemas y estrategias para envejecer en sociedad (Tesis doctoral inédita). Universidad Complutense de Madrid, Madrid, España.

Lebrusán, I. (2020). Las dificultades para habitar en la vejez. Documentación Social, 6. https:// www.documentacionsocial.es/6/a-fondo/las-dificultades-para-habitar-en-la-vejez

Ley 27/2011, de 1 de agosto, sobre actualización, adecuación y modernización del sistema de Seguridad Social. Boletin Oficial del Estado (BOE), n 184, de 02/08/2011. https://www. boe.es/buscar/act.php?id=BOE-A-2011-13242

Libman, K., Fields, D. \& Saegert, S. (2012). Housing and health: A social ecological perspective on the us foreclosure crisis. Housing, Theory and Society, 29(1), 1-24. https://doi.org/1 $0.1080 / 14036096.2012 .624881$ 
Módenes, J. A. (2017). La inseguridad residencial por problemas económicos en España comparada con el entorno europeo. Papers: Revista de Sociología, 102(4), 673-703. http://dx.doi.org/10.5565/rev/papers.2416

Módenes, J. A. \& López-Colás, J. (2014). Cambio demográfico reciente y vivienda en España: ¿hacia un nuevo sistema residencial? Revista Española de Investigaciones Sociológicas, 148, 103-134. http://dx.doi.org/10.5477/cis/reis.148.103

Monteverde, M., Tomas, S., Acosta, L. D. \& Garay, S. (2016). Envejecimiento poblacional y magnitud de la dependencia en Argentina y México: perspectiva comparada con España. Revista Latinoamericana de Población, 10(18), 135-154. https://doi. org/10.31406/relap2016.v10.i1.n18.6

Naciones Unidas, Departamento de Asuntos Económicos y Sociales. (2003). Informe sobre la situación social en el mundo 2003. Vulnerabilidad social: Fuentes y desafios. Naciones Unidas.

Naciones Unidas, Oficina del Alto Comisionado. (1976). Pacto Internacional de Derechos Económicos, Sociales y Culturales, Naciones Unidas (PIDESC). Serie de Tratados, vol. 993, p. 3, del 16 de diciembre de 1966, entrada en vigor el 03 de enero de 1976. https:// www.ohchr.org/sp/professionalinterest/pages/cescr.aspx

Navarro, C. (2002). Exclusión en vivienda y estado de salud: una estimación para el caso español. En IX Encuentro de Economía Pública, Hacienda y Medio Ambiente. Vigo, 7 y 8 de febrero. https://dialnet.unirioja.es/servlet/libro?codigo=397566

Navarro, C. (2006). La exclusión en vivienda en España: un análisis económico de su extensión, dinámica y efectos sobre el bienestar. Consejo Económico y Social de España.

Navarro, C. \& Ayala, L. (2008). Multidimensional housing deprivation indices with application to Spain. Applied Economics, 40(5), 597-611. https://doi. org/10.1080/00036840600722323

Office of Policy Development and Research (PD\&R). (2013). Measuring the costs and savings of aging in place. Evidence Matters. https://www.huduser.gov/portal/periodicals/em/ fall13/highlight $2 . \mathrm{html}$

Nolan, B. \& Winston, N. (2011). Dimensions of housing deprivation for older people in Ireland. Social Indicators Research, 104(3), 369-385. https://doi.org/10.1007/s11205010-9748-7

Oldman, C. (2003). Devicing, theorizing and self-justification a critique of independent living. CriticalSocialPolicy, 23(1), 44-62.https://doi.org/10.1177\%2F026101830302300103

Phillips, J. E., Ajrouch, K. J. \& Hillcoat-Nallétamby, S. (2010). Key concepts in social gerontology. Sage.

Pinzón-Pulido, S. A. (2016). Atención residencial vs. atención domiciliaria en la provisión de cuidados de larga duración a personas mayores en situación de dependencia (Tesis doctoral inédita). Universidad de Sevilla, Sevilla, España. https://idus.us.es/ handle/11441/44336

Pynoos, J., Nishita, C. \& Perelma, L. (2003). Advancements in the home modification field: A tribute to M. Powell Lawton. Journal of Housing for the Elderly, 17(1-2), 105-116. https://doi.org/10.1300/J081v17n01_08 
Redondo, N., Díaz Fernández, M., Llorente Marrón, M. del M., Garay, S., Guidotti González, C. A. \& Mendoza Villavicencio, L. M. (2015). El espacio residencial del cuidado de los adultos mayores en América Latina y Espańa. Notas de Población, 42(100), 223258. https://doi.org/10.18356/930eaddb-es

Rosow, I. (1974). Socialization to old age. University of California Press.

Rowles, G. (1978). Prisoners of space? Westview Press.

Saiegh, B. (2012). Relación entre las condiciones de la vivienda y mortalidad en la población española mayor de 65 años (Tesis doctoral inédita). Universidad Rey Juan Carlos, Madrid, España.

Şar, A. H., Göktürk, G. Y., Tura, G. \& Kazaz, N. (2012). Is the internet use an effective method to cope with elderly loneliness and decrease loneliness symptom? Procedia-Social and Behavioral Sciences, 55, 1053-1059. https://doi.org/10.1016/j.sbspro.2012.09.597

Zuluaga, M. C. Z. (2010). Depresión, calidad de vida y condiciones de la vivienda como predictores de la mortalidad en ancianos con insuficiencia cardiaca (Tesis doctoral inédita). Universidad Autónoma de Madrid, Madrid, España. https://repositorio. uam.es/bitstream/handle/10486/5193/33053_zuluaga_zuluaga_maria_clemencia. pdf? sequence $=1$ 